\title{
Transclavicular Osseous Sutures for the Treatment of Displaced Distal Clavicular Fractures in Children
}

\author{
Jörg Schilcher, Johan Scheer and Lars Adolfsson
}

\section{Linköping University Post Print}

\section{Tweet}

N.B.: When citing this work, cite the original article.

Original Publication:

Jörg Schilcher, Johan Scheer and Lars Adolfsson, Transclavicular Osseous Sutures for the Treatment of Displaced Distal Clavicular Fractures in Children, 2016, Journal of Orthopaedic Trauma, (30), 5, E181-E185.

http://dx.doi.org/10.1097/BOT.0000000000000527

Copyright: Lippincott, Williams \& Wilkins

http://www.lww.com/

Postprint available at: Linköping University Electronic Press

http://urn.kb.se/resolve?urn=urn:nbn:se:liu:diva-128739 
Osseous sutures for clavicular fractures in children

\section{Trans-clavicular osseous sutures for the treatment of}

\section{displaced distal clavicular fractures in children.}

Jörg Schilcher, PhD, Johan Scheer, PhD and Lars Adolfsson, Professor

All from the Department of Experimental and Clinical Medicine and Department of

Orthopedic Surgery, Faculty of Health Science, Linköping University, Linköping,

Sweden

Correspondence

Jörg Schilcher

Department of Experimental and Clinical Medicine

Faculty of Health Science

Linköping University

SE-581 85 Linköping

Sweden

E-mail: Jorg.schilcher@regionostergotland.se

Phone: +46101034312

Fax: +46101034305

\section{Conflicts of Interest and Source of Funding:}

No conflicts of interest to declare. The study was funded by Östergötland County Council, Sweden. 
Osseous sutures for clavicular fractures in children

\section{Summary}

We describe a novel surgical technique for the treatment of displaced distal clavicular fractures in children. These fractures are rare and recommendations on treatment vary. Conservative treatment might lead to persistent deformity and limitations of function. Previous reports of surgical treatment involve fracture fixation with K-wires. This requires a routine sequential reoperation to remove the implant and has been associated with serious complications in some patients.

The surgical technique described here is based on osseous sutures through the clavicular shaft and coracoclavicular ligaments and is found successful for the treatment of distal clavicular fractures in children and may also be feasible for true acromioclavicular dislocations. The main principle of the technique is a fixation of the displaced clavicle via trans-clavicular drill holes, against the intact inferior periosteal sleeve at the insertion of the coracoclavicular ligaments. No temporary K-wire fixation is needed. To date, we have treated 7 patients with this technique. All fractures healed uneventfully with an excellent functional result and without skeletal deformity. 
Osseous sutures for clavicular fractures in children

\section{Introduction}

Clavicular fractures in children are common injuries and constitute between 8 to 15\% of all pediatric fractures. ${ }^{(1-3)}$ Of these, 10 to $21 \%$ occur in the distal third of the bone. In the lateral epiphysis of the clavicle, ossification does not occur until the age of 18 years and the majority of injuries involve the metaphyseal-epiphyseal junction and not the acromioclavicular joint itself. In displaced distal clavicular injuries the thick periosteal sleeve remains partially intact, peeling off the fractured bone like the skin from a banana. ${ }^{(4)}$ The inferior part of the periosteum usually remains attached to the coracoclavicular ligaments and the diaphysis is displaced superiorly thus mimicking the radiographic appearance of acromioclavicular dislocations in adults ${ }^{(5)}$. The intact periosteum enables rapid healing and good remodeling of the injured bone, making surgical interventions unnecessary in the majority of cases ${ }^{(6,7)}$. However some patients will suffer persistent osseous deformity and limited function. ${ }^{(4,8-10)}$ The inferior periosteal sleeve and coracoclavicular ligaments often remain intact in distal clavicular fractures ${ }^{(4)}$ and appear to provide sufficient anchorage to keep the clavicular shaft reduced in its periosteal sleeve, maintaining stable fixation during fracture healing. Based on these conditions of the soft tissue envelope, the senior author (LA) has developed an osseous suture technique to treat these fractures without the use of K-wires. The aim of the present study was to describe the technique and report the results of 7 patients treated with this method.

\section{Patients}

Between 2005 and 2013, seven patients with displaced distal clavicular fractures were treated with open reduction and internal fixation using the described technique. There were two female and five male patients between 10 and 16 years of age. 
Osseous sutures for clavicular fractures in children

Following approval from the local committee for medical ethics (DNR: 2014/406-31), all patients were retrospectively identified from our orthopaedic surgical registry and offered a follow-up examination. All identified patients gave informed consent and attended a clinical and radiographic examination in the autumn of 2014, at a mean of 6.4 years after the operation (range 1-9 years). Standard x-rays in anterior and oblique views were taken. During clinical examination range of motion, patients's subjective assessment of function, and cosmesis was recorded and function was assessed using the Constant-Murley score. ${ }^{(11)}$ The x-rays were assessed and the fractures classified according to the classifications proposed by Dameron and Rockwood ${ }^{(9)}$ and Nenopolous et al ${ }^{(16)}$. The patients’ demographic data, fracture classification, fixation method, and outcomes are presented in the Table.

\section{Surgical technique}

All surgeries were performed on consecutive patients within our healthcare district by 5 different consultant surgeons. All patients were operated on within 10 days after the initial trauma. The treating physician judged the indication for surgery after physical examination and review of radiographs and was introduced to the technique by the senior author.

\section{Preparation}

All patients underwent general anesthesia and were washed and draped according to institutional standards. On the operating table the patients were kept in a beach-chair position and the table was positioned to facilitate intraoperative radiography.

\section{Skin incision and deep dissection}

The preferred skin incision was a roughly $4 \mathrm{~cm}$ sagittal incision at the level of the fracture line (Figure 1). Longitudinal skin incisions should be avoided due to cosmetic disadvantages. The incision was then carried through the subcutaneous tissue down to 
Osseous sutures for clavicular fractures in children

the superficial fascia overlying the trapezius muscle. The displaced clavicular shaft was found immediately subcutaneous while the fractured lateral end remained attached to the acromioclavicular joint capsule. If necessary the periosteum was incised slightly further to allow mobilization of the fragments. Some fibers of the trapezius muscle were found injured by the trauma in some of the patients. These were sutured at the end of the procedure.

\section{Fracture reduction and fixation}

The coracoid process was palpated through the intact inferior periosteum and the point where the coracoclavicular ligaments insert into the periosteal sleeve was marked with a sterile pen or suture. The medial clavicular fragment was reduced into the periosteal sleeve by lifting of the arm and shoulder girdle in a cranial direction and the fracture was then kept reduced with a clamp. Two $2 \mathrm{~mm}$ bicortical drill holes were drilled in the clavicular shaft exiting in the inferior cortex at the level of the coracoclavicular ligaments. A No. 2 braided and non-resorbable suture was passed from superior to inferior through the bicortical drill holes. The suture was secured in the inferior periosteum and through the coracoclavicular ligaments with a MasonAllen stitch. The suture was then passed from inferior to superior through the other drill hole. The threads were then tightened against the superior aspect of the clavicular shaft keeping it reduced to the ligaments and into the periosteal sleeve (Figure 2A). Subsequently one unicortical hole was drilled in the superior cortex of each fracture fragment. To enhance stability and to fine-tune reduction another No. 2 braided and non-resorbable suture was passed through the unicortical drill holes in the superior cortex of each fracture fragment (Figure 2B). The thread was then tightened and provided reduction and stability along the longitudinal axis of the clavicle. Finally, 
Osseous sutures for clavicular fractures in children

the periosteal sleeve was closed with No. 2-0 braided and resorbable sutures (Figure 2C).

Postoperative Management

Post-operatively a collar-cuff sling was used for three weeks. A physiotherapist instructed in active, unloaded movement exercises allowing flexion and abduction up to 90 degrees. After 3-4 weeks full range of motion was allowed and full use of the arm in every-day activities. Heavy load and contact sports was discouraged during a minimum of 8 weeks after the operation.

\section{Results}

All fractures healed uneventfully and reduction was maintained at early check-up visits. At an average follow-up period of 6 years (range 1-9 years) all patients reported good functional outcomes with excellent Constant-Murley scores and no remaining skeletal deformity. Final follow-up radiographs showed close to anatomic appearance of the clavicle and acromioclavicular joint in all cases (Figure 3 and 4 and Supplemental Digital Content 1).

Two patients complained of hypertrophic scar formation. In both cases a skin incision parallel to the lateral clavicle had been used instead of the standard sagittal incision. On physical examination none of the clavicles appeared shorter or displaced superiorly at the distal end and no instability of the lateral end of the clavicle was neither reported nor noted during arm movements. No hypertrophic callus formation, duplication of the clavicle or ossification of the coracoclavicular ligaments were seen.

\section{Discussion}


Osseous sutures for clavicular fractures in children

The trans-clavicular suture technique was developed to provide a surgical solution, without the need for temporary K-wire or cerclage wire fixation, in patients with severely displaced distal clavicular fractures suspected to result in a functional deficit or a poor cosmetic result if treated conservatively. All seven patients in the present series had an excellent functional and radiographic outcome at final follow-up.

True acromioclavicular joint dislocations rarely occur in children below the age of 13. ${ }^{(5)}$ The displacement is most often due to a lateral clavicular fracture, leaving the lateral clavicular fragment and epiphyseal growth plate in place. The capsule of the acromioclavicular joint remains undisturbed. Dameron and Rockwood ${ }^{(10)}$ have popularized a classification system for distal clavicular fractures in children similar to the classification system by Rockwood for acromioclavicular injuries in adults. ${ }^{(12)}$ Injuries are categorized into types I to VI with increasing numbers based on the severity and direction of displacement. Up to date it remains unclear, which of the fracture types benefit from reduction and surgical fixation to improve outcome. Although these fractures usually do not affect the acromioclavicular joint, the surrounding injury of the musculature appears similar to that occurring in acromioclavicular joint dislocations in adults. In these injuries there seems to be agreement that surgery is indicated when the soft tissue envelope is severely damaged. ${ }^{(13)}$ For that reason, in the present series surgical intervention was recommended for all type V fractures but also for three patients with type III fractures in whom the displacement was apparent on the exterior and a soft tissue involvement similar to a type V lesion was suspected clinically. Also, these patients were older, having less potential for remodeling. 
Osseous sutures for clavicular fractures in children

Furthermore, all fractures in our series were classified according to Nenopolous et al, ${ }^{(14)}$ a modern classification system for distal clavicular fractures in children. All injuries in our series were graded as group $2 b$, 3b or 5 for which these authors recommend surgery. Group $2 \mathrm{~b}$ injuries are transverse, displaced fractures, grade 3b injuries are displaced oblique fractures and surgery is recommended when the displacement exceeds the thickness of 2 cortices. Group 5 injuries are true acromioclavicular dislocations.

In general however, the acromioclavicular ligaments, the acromioclavicular joint and most of the periosteal sleeve is intact in distal clavicular injuries providing exceptional potential for growth and remodeling. It remains unclear if these patients in our series profited from the surgical intervention since several other authors have stated that the majority of patients with fracture types I, II and III can be treated nonsurgical with a good functional and cosmetic result. ${ }^{(6-8,10,15)}$ For the displaced fracture types IV, V and VI some authors suggest that most patients will regain full function without cosmetic deficits ${ }^{(6,7)}$. Other authors recommend that patients with grossly displaced fractures or fixed displacement should be treated with reduction and surgical fixation to prevent definitive deformity. ${ }^{(4,8-10)}$ With increasing age, and decreasing capacity to remodel a deformity the indication for surgical reduction and fixation becomes stronger. ${ }^{(5)}$ Previously described treatment options for displaced fractures involve percutaneous fixation with K-wires and suture repair of the superior periosteal sleeve or more extensive soft tissue repairs without the use of hardware. ${ }^{(4,}$ 16) However, severe complications caused by the migration of K-wires to the lungs and spinal canal have been reported. ${ }^{(17-19)}$ Furthermore, K-wire fixation is associated with compulsory reoperations to remove the implant. This might make surgical 
Osseous sutures for clavicular fractures in children

fixation of displaced fractures less attractive for surgeons, patients and their caretakers. Therefore the trans-clavicular suture technique described here might be a more attractive alternative in some cases. The method is a simplified modification of a previously described technique, ${ }^{(4,16)}$ aiming at using the strong point of fixation provided by the coraco-clavicular ligaments and without the use of cerclage wires. ${ }^{(20)}$ Recently a similar technique, using sutures around the coracoid process, was reported to provide successful fixation in displaced type II distal clavicular fractures in adults. ${ }^{(21)}$

In our series 5 different surgeons performed the surgeries on the 7 patients. This might be regarded as disadvantageous because of the very low volume per surgeon. However, we believe it emphasizes the simplicity of the technique ensuring good results even when performed seldom. Surgeries performed via a longitudinal incision had a higher likelihood to lead to unsatisfactory cosmetic results due to the formation of hypertrophic scar tissue. The extension of the incision medially towards skin areas prone to the formation of hypertrophic scars and keloids might be the reason for this. Moreover the sagittal incision should be preferred because it provides better access for suture placement and follows the course of Langer's skin lines (Figure 1), which too is cosmetically favorable. Also the risk of injuring the lateral supraclavicular nerve branch, providing skin sensation caudal to the clavicle is lower. In adults the course of this nerve is highly variable but has a consistent safe zone up to $1.9 \mathrm{~cm}$ medial to the acromioclavicular joint. ${ }^{(22)}$ We therefore recommend the sagittal skin incision as the standard approach. The lack of a conservatively treated control group makes it impossible to evaluate the effect of the surgical procedure on the outcome. At the current level of evidence, the trans-clavicular suture technique is a safe surgical 
Osseous sutures for clavicular fractures in children

treatment option for patients with severely displaced distal clavicular fractures where surgical reduction and fixation deems indicated to improve outcome. 
Osseous sutures for clavicular fractures in children

\section{References}

1. Landin LA. Fracture patterns in children. Analysis of 8,682 fractures with special reference to incidence, etiology and secular changes in a Swedish urban population 1950-1979. Acta Orthop Scand Suppl. 1983;202:1-109.

2. Lichtenberg RP. A study of 2,532 fractures in children. Am J Surg. 1954;87:330-338.

3. Nordqvist A, Petersson C. The incidence of fractures of the clavicle. Clin Orthop Relat Res. 1994:127-132.

4. Falstie-Jensen S, Mikkelsen P. Pseudodislocation of the acromioclavicular joint. J Bone Joint Surg Br. 1982;64:368-369.

5. Eidman DK, Siff SJ, Tullos HS. Acromioclavicular lesions in children. Am J Sports Med. 1981;9:150-154.

6. Black GB, McPherson JA, Reed MH. Traumatic pseudodislocation of the acromioclavicular joint in children. A fifteen year review. Am J Sports Med. 1991;19:644-646.

7. Havranek P. Injuries of distal clavicular physis in children. J Pediatr Orthop. 1989;9:213-215.

8. Ogden JA. Distal clavicular physeal injury. Clin Orthop Relat Res. 1984:6873.

9. Bakalim G, Wilppula E. Surgical or conservative treatment of total dislocation of the acromioclavicular joint. Acta Chir Scand. 1975;141:43-47.

10. Dameron T, Rockwood C. Fractures in children. In: Rockwood C, Wilkins K, King R, eds. Philadelphia: JB Lippincott; 1984:624-653.

11. Constant CR, Murley AHG. A clinical method of functional assessment of the shoulder. Clin Orthop Relat Res. 1987;No. 214:160-164.

12. Williams G, Nguyen V, Rockwood C. Classification and radiographic analysis of acromioclavicular dislocations. Appl Radiol. 1989;Feb:29-34.

13. Bradley JP, Elkousy H. Decision making: operative versus nonoperative treatment of acromioclavicular joint injuries. Clin Sports Med. 2003;22:277-290. 14. Nenopoulos SP, Gigis IP, Chytas AA, et al. Outcome of distal clavicular fracture separations and dislocations in immature skeleton. Injury. 2011;42:376380.

15. Masnovi ME, Mehlman CT, Eismann EA, et al. Pediatric refracture rates after angulated and completely displaced clavicle shaft fractures. J Orthop Trauma. 2014;28:648-652.

16. Gronmark T. Surgical treatment of acromioclavicular dislocation. Acta Orthop Scand. 1976;47:308-310.

17. Bennis S, Scarone P, Lepeintre JF, et al. Asymptomatic spinal canal migration of clavicular K-wire at the cervicothoracic junction. Orthopedics. 2008;31.

18. Hegemann S, Kleining R, Schindler HG, et al. Kirschner wire migration in the contralateral lung after osteosynthesis of a clavicular fracture. Unfallchirurg. 2005;108:991-993.

19. Mamane W, Breitel D, Lenoir T, et al. Spinal migration of a Kirschner wire after surgery for clavicular nonunion. A case report and review of the literature. Chir Main. 2009;28:367-369. 
Osseous sutures for clavicular fractures in children

20. Montgomery SP, Loyd RD. Avulsion fracture of the coracoid epiphysis with acromioclavicular separation. Report of two cases in adolescents and review of the literature. J Bone Joint Surg Am. 1977;59:963-965.

21. Duralde XA, Pennington SD, Murray DH. Interfragmentary suture fixation for displaced acute type II distal clavicle fractures. J Orthop Trauma. 2014;28:653-658.

22. Nathe T, Tseng S, Yoo B. The anatomy of the supraclavicular nerve during surgical approach to the clavicular shaft. Clin Orthop Relat Res. 2011;469:890894.

\section{Figure Legend}

Figure 1. Sagittal incision demonstrated on a right shoulder. The recommended incision runs over the distal clavicle along the lines of Langer and at the tip of the coracoid process. Apart from improved cosmesis and optimal surgical access for the repair, the incision is located at a distance from the supraclavicular nerves. ${ }^{(22)}$ NZ=Nerve Zone.

Figure 2. Surgical technique: Two holes are drilled in the medial fragment of the clavicle. Sutures are passed through and attached to the intact inferior periosteum with a Mason-Allen stitch (A). The sutures are then tightened against the superior aspect of the clavicular shaft and locked with a standard surgical knot. This maneuver reduces the clavicle back into the periosteal sleeve $(\mathbf{B})$. Sutures are then passed through unicortical drill holes in the superior cortex of each fracture fragment and tightened. This provides reduction and stability along the longitudinal axis of the clavicle (C). The superior periosteal sleeve is then repaired.

Figure 3. Twelve-year-old patient with displaced lateral clavicular fracture (A) treated with the trans-clavicular suture technique $(\mathbf{B})$. At follow-up, 9 years after surgery the fracture had remodeled adequately $(\mathbf{C})$.

Figure 4. Twelve-year-old patient with displaced lateral clavicular fracture (A) treated with the trans-clavicular suture technique $(\mathbf{B})$. At follow-up, 1 year after surgery the fracture had remodeled adequately $(\mathbf{C})$. 
Osseous sutures for clavicular fractures in children

\section{Online supplementary material}

Online supplementary material 1 . Thirteen-year-old patient with displaced lateral clavicular fracture (A) treated with the trans-clavicular suture technique (B). At follow-up, 8 years after surgery the fracture had remodeled adequately (C). 
Osseous sutures for clavicular fractures in children 
Table. Patient Demographics, Fracture Classification, Treatment and Outcomes

\begin{tabular}{|c|c|c|c|c|c|c|c|c|}
\hline Case No. & $\begin{array}{l}\text { Sex } \\
(M / F)\end{array}$ & $\begin{array}{l}\text { Age at } \\
\text { injury } \\
\text { (Years) }\end{array}$ & $\begin{array}{l}\text { Follow-up } \\
\text { (Years) }\end{array}$ & $\begin{array}{l}\text { Dameron and } \\
\text { Rockwoods }^{(9)} \\
\text { classification }\end{array}$ & $\begin{array}{l}\text { Nenopoulos }^{(16)} \\
\text { classification }\end{array}$ & $\begin{array}{l}\text { Side } \\
\text { (D/ND) }\end{array}$ & $\begin{array}{l}\text { Constant-Murley } \\
\text { score* } \\
\text { (injured side) }\end{array}$ & $\begin{array}{l}\text { Constant-Murley } \\
\text { score* } \\
\text { (uninjured side) }\end{array}$ \\
\hline 1 & $\mathrm{M}$ & 12 & 9 & V & $2 b$ & $\mathrm{D}$ & 97 & 96 \\
\hline 2 & $\mathrm{M}$ & 13 & 8 & V & $2 b$ & $\mathrm{D}$ & 95 & 95 \\
\hline 3 & $\mathrm{~F}$ & 16 & 8 & III & $2 b$ & $\mathrm{D}$ & 89 & 89 \\
\hline 4 & $\mathrm{M}$ & 10 & 7 & V & $3 b$ & $\mathrm{D}$ & 95 & 93 \\
\hline 5 & $\mathrm{M}$ & 13 & 7 & III & 5 & ND & 87 & 91 \\
\hline 6 & $\mathrm{~F}$ & 14 & 5 & III & $2 b$ & $\mathrm{D}$ & 91 & 91 \\
\hline 7 & $\mathrm{M}$ & 12 & 1 & V & $2 b$ & ND & 90 & 91 \\
\hline
\end{tabular}

D: Dominant. ND: Non dominant. * Constant-Murley score at final follow up. 


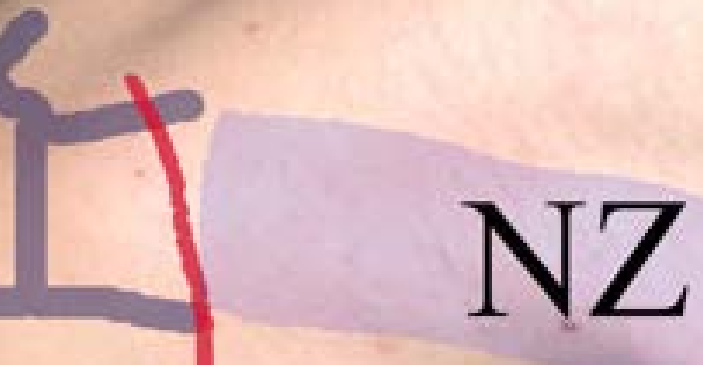



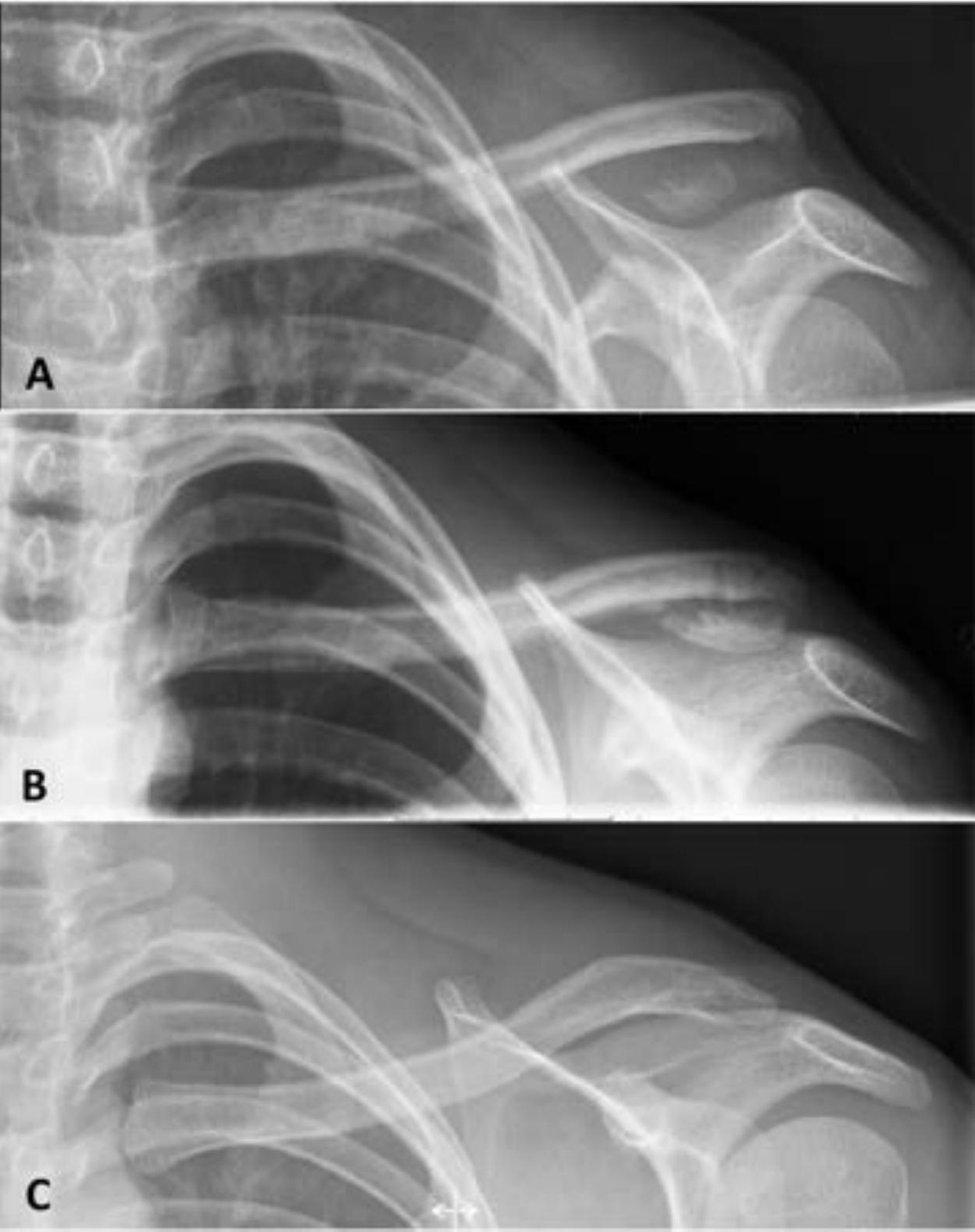
A

B

$\frac{x O}{D X}$

C 


\section{A}

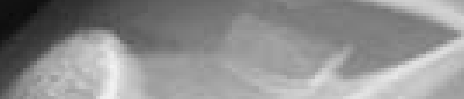

B

\section{$x 0$}

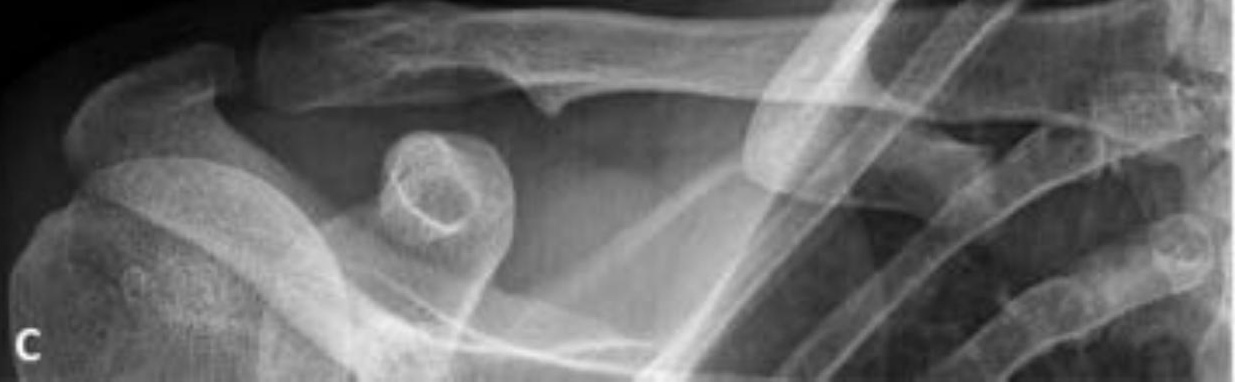

